causes were vascular episodes (5/11) and infection of central nervous system (3/11), while others, including the head trauma, accounted for one case respectively. Most usual time of onset was during first trimester (6/11), while timing in other five children was distributed quite equally until the age of two years of life.

Postneonatal cerebral palsy is rare and possibly unrecognised form of cerebral palsy, in our Register, as well as common SCPE register (proportion of $7.69 \%$ of total CP population; prevalence of 1.26/10000). Recognition of this state is important since it is partly preventable, epidemiologically relevant in large scale studies, but most importantly, on individual level, for planning and establishing the special care each CP patient should have.

\section{NEUROSURGICAL TREATMENT OF INTRACTABLE EPILEPSIES DUE TO FOCAL CORTICAL DYSPLASIA}

Sanja Marinković* Vlasta Đuranović. General county hospital Vinkovci

10.1136/archdischild-2021-europaediatrics.401

Focal cortical dysplasia is the most common cause of focal intractable epilepsy in children. According to the ILAE classification there are 3 basic forms of FCD. As diagnostic methods become more sophisticated more often FCD's are recognized as the cause of epilepsy. With the advancement of neurosurgical techniques, surgical procedures are becoming an important element of treating epilepsies.

We would like to represent 2 of our patients with intractable epilepsy due to FCD, who have undergone a neurosurgical evaluation and treatment with excellent results - their epilepsy is cured.

First girl was born from uneventful pregnancy with normal development until the age of 11 months, when seizures began. Her brain CT was normal. As seizures with focal onset repeated, and EEG showed focal changes on the right, anticonvulsive therapy was administrated. Next two years she had just one episode of febrile seizure during illness. Brain MRI done at the age of

4 years was normal. In the next period she had focal seizures with motoric component every couple of months. AET was changed but without clinical improvement. Brian MRI at the age of $9.5 \mathrm{y}$ was normal, but her epilepsy worsened, her focal seizures were often in duration of status epilepticus, so she was referred to Children's hospital Zagreb. Brain MRI done with special sequences shown increased cortical thickness in precentral and postcentral gyri, diagnosis of FCD was made, she was presented to neurosurgical team and after evaluation she was operated. After curative resection, diagnosis of FCD type III B with ganglioma gradus II was made.

After the operation she is seizure free with left sided hemiparesis. Her academic achievements are good.

Second girl was born from uneventful pregnancy and reached her early milestones. At the age of 9 months, she had complex febrile seizure, that repeated at the age of 13 months. Seizures exacerbated during first year of life, so she had many febrile and afebrile seizure episodes, often with duration of epileptic status, without response to antiepileptic therapy.
Neurological decline was evident with left-sided hemiparesis. Brain MRI showed increased cortical thickness on the right precentral gyri and she was referred to neurosurgical evaluation. She was operated and resection of lesion was done, she is now seizure-free, with left-sided hemiparesis.

The lesion showed characteristics of FCD II $b$.

Conclusion Focal epileptogenic lesions can cause generalized epilepsy syndromes in children. The aim of neurosurgical interventions is to eliminate the epileptogenic lesion.

\section{SLEEP DISTURBANCES IN CHILDREN WITH AUTISM SPECTRUM DISORDERS}

Romana Gjergja Juraski*, Matilda Kovac Sizgoric, Mirta Loncar, Ivana Marusic, Marija Milos, Hrvoje Pap, Ines Kunic. Children's Hospital Srebrnjak

\subsection{6/archdischild-2021-europaediatrics.402}

Autism Spectrum Disorders (ASD) are characterized by persistent impairments in reciprocal social interaction and communication. Sleep problems in ASD have significant impact on daily function, social interaction, academic achievements resulting in increased parental sleep disruption and stress.

The genetic factors underlying autism interact in the genesis of the higher percentage of sleep disturbances in children's autism.

During the 4-year-period 64 ASD children were refferred to our Sleep lab and performed an overnight polysomnography (50 males, 14 females, average age

6.76 years, range 2.5-17.5). Inclusion criteria were a diagnosis of ASD using the Diagnostic and Statistical Manual of Mental Disorders IV-R/5 criteria. Exclusion criteria were secondary ASD (e.g. associated with fragile X syndrome, Rett syndrome, Down syndrome etc). Patients with severe physical disability or severe allergy were also excluded from the study.

Polysomnographic parameters included: EMG, EOG, 6-16 channel EEG, nasal pressure, oro-nasal thermistor, chest and abdominal belts for respiratory effort, oximetry with waveform, snoring microphone, EKG, body position, video and audio. Parents filled in the sleep questionnaire.

The analysis revealed that most of the sleep disturbancies included decreased REM quantity, increased undifferentiated sleep, immature organization of eye movements into discrete bursts, decreased time in bed, total sleep time, REM sleep latency, and increased proportion of NREM1 sleep. The sleep fragmentation was higher in children with psychomotoric delay.There was the higher percentage of sleep disruption in the subgroup with focal or/and generalized discharges on EEG, with or without epilepsy.

If treated, the mostly used antiepileptic drugs were valproic acid and levetiracetam.

Polysomnography was useful not only in identifying sleep disorders, but also in demonstrating seizure discharges. Treatment strategies along with limited regulated pharmacotherapy could help improve daily function, social interaction and the quality of life in ASD children and have a beneficial impact on the family. Implementation of bedtime routines and sleepwise approach is still the mainstay of behavioral management in children with autism. 